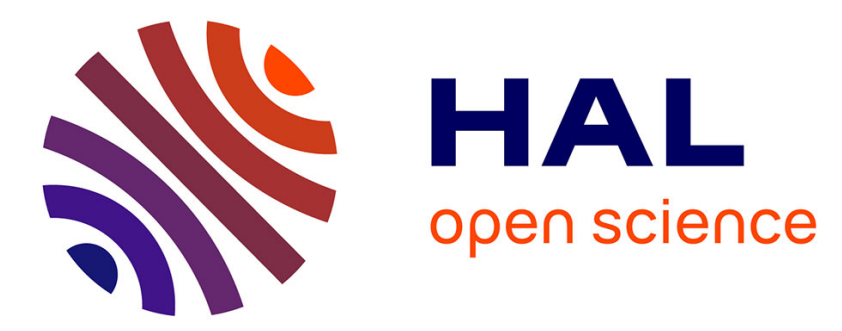

\title{
Asymptotic Limits of a New Type of Maximization Recurrence with an Application to Bioinformatics
}

Kun-Mao Chao, An-Chiang Chu, Jesper Jansson, Richard S. Lemence, Alban Mancheron

\section{- To cite this version:}

Kun-Mao Chao, An-Chiang Chu, Jesper Jansson, Richard S. Lemence, Alban Mancheron. Asymptotic Limits of a New Type of Maximization Recurrence with an Application to Bioinformatics. TAMC'12: 9th Annual Conference on Theory and Applications of Models of Computation, May 2012, Beijing, China. pp.622, 10.1007/978-3-642-29952-0 . lirmm-00717670

\section{HAL Id: lirmm-00717670 https://hal-lirmm.ccsd.cnrs.fr/lirmm-00717670}

Submitted on 13 Jul 2012

HAL is a multi-disciplinary open access archive for the deposit and dissemination of scientific research documents, whether they are published or not. The documents may come from teaching and research institutions in France or abroad, or from public or private research centers.
L'archive ouverte pluridisciplinaire HAL, est destinée au dépôt et à la diffusion de documents scientifiques de niveau recherche, publiés ou non, émanant des établissements d'enseignement et de recherche français ou étrangers, des laboratoires publics ou privés. 


\title{
Asymptotic Limits of a New Type of Maximization Recurrence with an Application to Bioinformatics
}

\author{
Kun-Mao Chao ${ }^{1}$, An-Chiang Chu ${ }^{1}$, Jesper Jansson ${ }^{2}$, Richard S. Lemence ${ }^{2,3}$, \\ and Alban Mancheron ${ }^{4}$ \\ 1 Department of Computer Science and Information Engineering, \\ National Taiwan University, Taipei, Taiwan 106 \\ kmchao@csie.ntu.edu.tw, anchiang@gmail.com \\ 2 Ochanomizu University, 2-1-1 Otsuka, Bunkyo-ku, Tokyo 112-8610, Japan \\ Jesper.Jansson@ocha.ac.jp, rslemence@gmail.com \\ 3 Institute of Mathematics, College of Science, University of the Philippines, \\ Diliman, Quezon City, 1101 Philippines \\ 4 Université Montpellier 2, LIRMM/CNRS, 161 rue Ada, \\ 34095 Montpellier Cedex 5, France \\ alban.mancheron@lirmm.fr
}

\begin{abstract}
We study the asymptotic behavior of a new type of maximization recurrence, defined as follows. Let $k$ be a positive integer and $p_{k}(x)$ a polynomial of degree $k$ satisfying $p_{k}(0)=0$. Define $A_{0}=0$ and for $n \geq 1$, let $A_{n}=\max _{0 \leq i<n}\left\{A_{i}+n^{k} p_{k}\left(\frac{i}{n}\right)\right\}$. We prove that $\lim _{n \rightarrow \infty} \frac{A_{n}}{n^{k}}=$ $\sup \left\{\frac{p_{k}(x)}{1-x^{k}}: 0 \leq x<1\right\}$. We also consider two closely related maximization recurrences $S_{n}$ and $S_{n}^{\prime}$, defined as $S_{0}=S_{0}^{\prime}=0$, and for $n \geq 1$, $S_{n}=\max _{0 \leq i<n}\left\{S_{i}+\frac{i(n-i)(n-i-1)}{2}\right\}$ and $S_{n}^{\prime}=\max _{0 \leq i<n}\left\{S_{i}^{\prime}+\left(\begin{array}{c}n-\bar{i} \\ 3\end{array}\right)+\right.$ $\left.2 i\left(\begin{array}{c}n-i \\ 2\end{array}\right)+(n-i)\left(\begin{array}{c}i \\ 2\end{array}\right)\right\}$. We prove that $\lim _{n \rightarrow \infty} \frac{S_{n}}{n^{3}}=\frac{2 \sqrt{3}-3}{6} \approx 0.077350 \ldots$ and $\lim _{n \rightarrow \infty} \frac{S_{n}^{\prime}}{3\left(\begin{array}{c}n \\ 3\end{array}\right)}=\frac{2(\sqrt{3}-1)}{3} \approx 0.488033 \ldots$, resolving an open problem from Bioinformatics about rooted triplets consistency in phylogenetic networks.
\end{abstract}

\section{Introduction}

A recurrence relation (or recurrence, for short) is an equation of the form $T_{n}=$ $f\left(T_{n-1}, T_{n-2}, \ldots, T_{0}, n\right)$, where $f$ is a specified function and $n$ is an unspecified positive integer, along with the values $T_{0}, T_{1}, \ldots, T_{m}$ for some finite, non-negative integer $m$. Intuitively, a recurrence describes how the value of $T_{n}$ for any $n$ depends on $n$ and the values of the elements in the sequence $T_{0}, T_{1}, \ldots, T_{n-1}$.

Recurrences are central to the analysis of algorithms [3]. In particular, when recursion is involved, the worst-case running time $T_{n}$ of an algorithm for an input of size $n$ can often be expressed in terms of $T_{n_{1}}, T_{n_{2}}, \ldots, T_{n_{k}}$, where $n_{1}, n_{2}, \ldots, n_{k}<n$, which naturally yields a recurrence. It can be argued that recurrences are as important to Theoretical Computer Science as differential equations are to Physics. Over the years, elegant techniques for solving various types 
of linear recurrences (i.e., recurrences for which the function $f$ mentioned above is a linear function) have been developed, and are now part of most standard undergraduate and graduate algorithm theory courses [3]. However, much less is known about how to solve nonlinear recurrences, and no general technique that works for all types of nonlinear recurrences exists. Instead, people have focused on asymptotically bounding the values of $T_{n}$ as $n \rightarrow \infty$ for various special cases such as minimization recurrences of the form $T_{n}=\min _{1 \leq i<n}\left\{T_{i}+T_{n-i}\right\}+g(n)$, where $g$ is some auxiliary function, and maximization recurrences that use the max-function [5912 1315]. Interestingly, such recurrences have shown up in many different problems concerning random trees, Huffman coding, binomial group testing, dynamic programming, dichotomous search problems, the design of electrical circuits, binary search trees, quicksort, parallel divide-and-conquer algorithms, computational geometry, and tree-drawing.

In this paper, we contribute to the existing repertoire of tools for analyzing nonlinear recurrences. To be precise, we develop a technique for bounding the asymptotic behavior of a new type of maximization recurrence, defined as follows. Let $k$ be a positive integer and $p_{k}(x)$ a polynomial of degree $k$ satisfying $p_{k}(0)=$ 0 . Define $A_{0}=0$ and for $n \geq 1$, let

$$
A_{n}=\max _{0 \leq i<n}\left\{A_{i}+n^{k} p_{k}\left(\frac{i}{n}\right)\right\}
$$

We also consider two closely related maximization recurrences $S_{n}$ and $S_{n}^{\prime}$, defined as $S_{0}=S_{0}^{\prime}=0$, and for $n \geq 1$,

$$
S_{n}=\max _{0 \leq i<n}\left\{S_{i}+\frac{i(n-i)(n-i-1)}{2}\right\}
$$

and

$$
S_{n}^{\prime}=\max _{0 \leq i<n}\left\{S_{i}^{\prime}+\left(\begin{array}{c}
n-i \\
3
\end{array}\right)+2 i\left(\begin{array}{c}
n-i \\
2
\end{array}\right)+(n-i)\left(\begin{array}{l}
i \\
2
\end{array}\right)\right\}
$$

where $\left(\begin{array}{l}x \\ y\end{array}\right)=0$ if $x<y$. (At this point, the reader may like to verify that some consecutive values of $S_{n}^{\prime}$ are: $S_{0}^{\prime}=0, S_{1}^{\prime}=0, S_{2}^{\prime}=0, S_{3}^{\prime}=2, S_{4}^{\prime}=7, S_{5}^{\prime}=16$, $S_{6}^{\prime}=32, S_{7}^{\prime}=55, S_{8}^{\prime}=87, S_{9}^{\prime}=130, S_{10}^{\prime}=184, \ldots$, and this sequence does not appear to follow any regular pattern.)

Below, we derive non-trivial, constant values of the expressions $\lim _{n \rightarrow \infty} A_{n} / n^{k}$, $\lim _{n \rightarrow \infty} S_{n} / n^{3}$, and $\lim _{n \rightarrow \infty} S_{n}^{\prime} / 3\left(\begin{array}{l}n \\ 3\end{array}\right)$.

\subsection{Motivation}

Our motivation for studying the maximization recurrences in this paper originates from a combinatorial problem in Bioinformatics related to phylogenetic networks and rooted triplets consistency. This subsection describes the background; for further technical details, see [2] and [11.

One of the many objectives of Bioinformatics is to develop new concepts and tools that can help researchers visualize the evolutionary history of a set 
of species. Traditionally, phylogenetic trees (rooted, unordered, distinctly leaflabeled trees in which every internal node has at least two children) have been used for this purpose 4. As might be expected, it is computationally prohibitive in general to infer a reliable phylogenetic tree for a large set of species directly. A promising alternative is the supertree approach 18] which first infers highly accurate phylogenetic trees for many small, overlapping subsets of the species and then applies a combinatorial algorithm to merge them into a single phylogenetic tree. One variant of the supertree approach takes as input a set $\mathcal{R}$ of rooted triplets (binary phylogenetic trees with exactly three leaves each) whose leaf label sets overlap, and tries to construct a phylogenetic tree that is consistent with the maximum possible number of rooted triplets from $\mathcal{R}$, where a rooted triplet $t$ is said to be consistent with a phylogenetic tree $T$ if $t$ is an embedded subtree of $T$. Gassieniec et al. [6] presented a polynomial-time algorithm that outputs a phylogenetic tree which is consistent with at least $1 / 3$ of the rooted triplets in any input set $\mathcal{R}$, and also showed that for a particular set $\mathcal{R}$ of rooted triplets, no phylogenetic tree can be consistent with more than $1 / 3$ of its elements (to see this, just take the set $\mathcal{R}_{n}$ of all $3\left(\begin{array}{l}n \\ 3\end{array}\right)$ rooted triplets over a fixed leaf label set of cardinality $n$, for any $n \geq 3$ ). In this sense, the algorithm of Gasieniec et al. [6] is worst-case optimal for phylogenetic trees.

Due to certain evolutionary events such as hybridization that sometimes occur in nature, not all evolution is treelike. Therefore, the phylogenetic tree model was recently extended to phylogenetic networks that permit nodes to have more than one parent (see, e.g., the surveys in [1014]). One important special type of phylogenetic network, introduced by Wang et al. [16] and later termed "galledtree" by Gusfield et al. [7, requires all cycles in the underlying undirected graph to be node-disjoint. (Galled-trees are also known in the literature as "level-1 networks" [101114.) Obviously, galled-trees can express more complicated evolutionary relationships than phylogenetic trees. To measure how much more powerful galled-trees really are, we can compare the optimal $1 / 3$ bound stated above for phylogenetic trees to the corresponding bound for galled-trees, and this leads to the recurrence $S_{n}^{\prime}$ studied in the present paper. More precisely, Jansson et al. 11] proved that for any $n \geq 3$, no galled-tree can be consistent with more than a fraction of $S_{n}^{\prime} / 3\left(\begin{array}{l}n \\ 3\end{array}\right)$ of the elements in the set $\mathcal{R}_{n}$ of all rooted triplets over a fixed leaf label set of cardinality $n$. Later, Byrka et al. [2] gave a polynomial-time algorithm that constructs a galled-tree consistent with at least $S_{n}^{\prime} / 3\left(\begin{array}{l}n \\ 3\end{array}\right)$ of the rooted triplets in any input set $\mathcal{R}$.

Jansson et al. 11] showed that for large enough values of $n$, it holds that $S_{n}^{\prime} / 3\left(\begin{array}{l}n \\ 3\end{array}\right)<0.4883$. On the other hand, Byrka et al. [2] proved that $S_{n}^{\prime} / 3\left(\begin{array}{l}n \\ 3\end{array}\right)>$ 0.4800 for all $n$. However, both groups of authors were unable to derive tight asymptotic bounds on $S_{n}^{\prime} / 3\left(\begin{array}{l}n \\ 3\end{array}\right)$, and this has been one of the remaining open problems for galled-trees. Computations have suggested that $S_{n}^{\prime} / 3\left(\begin{array}{l}n \\ 3\end{array}\right)$ is closer to the upper bound 0.4883 than the lower bound 0.4800 , and indeed, we settle the issue in Section 3 by proving that $\lim _{n \rightarrow \infty} \frac{S_{n}^{\prime}}{3\left(\begin{array}{c}n \\ 3\end{array}\right)}=\frac{2(\sqrt{3}-1)}{3} \approx 0.488033 \ldots$ Observe that this improves the 5/12-ratio mentioned on p. 311 of 10 and the $48 \%$-ratio mentioned on p. 135 of [14]. 
The other two recurrences introduced in this paper, $S_{n}$ and $A_{n}$, were studied because of their connections to $S_{n}^{\prime}$. As shown in Lemma 2 in Section 2 below, the bound for $S_{n}^{\prime} / 3\left(\begin{array}{l}n \\ 3\end{array}\right)$ follows immediately from the bound for $S_{n} / n^{3}$, which is slightly easier to compute. $A_{n}$ is a special case of a generalization of $S_{n}$.

\section{$1.2 \quad$ Related Work}

The appearance of nonlinear recurrence relations eluding exact solutions in diverse fields of study has motivated many previous papers, including [5 912 13 15], to investigate their asymptotic properties on a case-by-case basis. For example, Fredman and Knuth [5] considered minimization recurrences of the form $T_{n}=\min _{1 \leq i<n}\left\{a \cdot T_{i}+b \cdot T_{n-i}\right\}+g(n)$, and Kapoor and Reingold [12] extended their results and also studied analogous maximization recurrences. In [13, Li and Reingold considered exact solutions and upper bounds for a special type of recurrence of the form $T_{n}=\max _{1 \leq i<n}\left\{T_{i}+T_{n-i}+\min \{g(i), g(n-i)\}\right\}$ involving minimization and maximization simultaneously, and in [9, Hwang and Tsai derived asymptotic approximations of this recurrence for more general auxiliary functions $g$. Saha and Wagh 15 studied a recurrence of the form $T_{n}=\min _{1 \leq i<n}\left\{\max \left\{T_{i}+a \cdot i, T_{n-i}\right\}+b\right\}$. Nevertheless, due to the irregular and often unpredictable behavior of nonlinear recurrences, general techniques for analyzing them still seem far from reach.

\subsection{Main Results and Organization of the Paper}

We establish the relationships among the three recurrences $A_{n}, S_{n}$, and $S_{n}^{\prime}$ in Section 2. Then, in Section 3. we prove that $\lim _{n \rightarrow \infty} \frac{S_{n}}{n^{3}}=\frac{2 \sqrt{3}-3}{6} \approx 0.077350 \ldots$ and that $\lim _{n \rightarrow \infty} \frac{S_{n}^{\prime}}{3\left(\begin{array}{c}n \\ 3\end{array}\right)}=\frac{2(\sqrt{3}-1)}{3} \approx 0.488033 \ldots$ Next, in Section 4 , we consider the ratio $A_{n} / n^{k}$. We show that $\lim _{n \rightarrow \infty} \frac{A_{n}}{n^{k}}=\sup \left\{\frac{p_{k}(x)}{1-x^{k}}: 0 \leq x<1\right\}$. Finally, Section 5 discusses generalizations of our techniques and an open problem.

\section{Preliminaries}

The two recurrences $S_{n}$ and $S_{n}^{\prime}$ are related as follows.

Lemma 1. For all $n \geq 0$, it holds that $S_{n}=S_{n}^{\prime}-\left(\begin{array}{l}n \\ 3\end{array}\right)$.

Proof. By induction on $n$. For $n=0$, we have $S_{0}=S_{0}^{\prime}=0$.

Next, suppose that $S_{k}=S_{k}^{\prime}-\left(\begin{array}{l}k \\ 3\end{array}\right)$ for all $k<n$. Then, since $\left(\begin{array}{c}n \\ 3\end{array}\right)=\left(\begin{array}{c}n-i \\ 3\end{array}\right)+$ $i\left(\begin{array}{c}n-i \\ 2\end{array}\right)+(n-i)\left(\begin{array}{l}i \\ 2\end{array}\right)+\left(\begin{array}{l}i \\ 3\end{array}\right)$ for every $0 \leq i<n$, we can rewrite $S_{n}^{\prime}$ as $S_{n}^{\prime}=\left(\begin{array}{c}n \\ 3\end{array}\right)+$ $\max _{0 \leq i<n}\left\{i\left(\begin{array}{c}n-i \\ 2\end{array}\right)+S_{i}^{\prime}-\left(\begin{array}{l}i \\ 3\end{array}\right)\right\}$. By the induction hypothesis: $S_{n}^{\prime}-\left(\begin{array}{l}n \\ 3\end{array}\right)=\max _{0 \leq i<n}\left\{i\left(\begin{array}{c}n-i \\ 2\end{array}\right)+\right.$ $\left.S_{i}^{\prime}-\left(\begin{array}{c}i \\ 3\end{array}\right)\right\}=\max _{0 \leq i<n}\left\{i\left(\begin{array}{c}n-i \\ 2\end{array}\right)+S_{i}\right\}=S_{n}$.

Lemma 2. $\lim _{n \rightarrow \infty} \frac{S_{n}^{\prime}}{3\left(\begin{array}{c}n \\ 3\end{array}\right)}=\lim _{n \rightarrow \infty} \frac{2 S_{n}}{n^{3}}+\frac{1}{3}$. 
Proof. From Lemma 1, we have $\lim _{n \rightarrow \infty} \frac{S_{n}^{\prime}}{3\left(\begin{array}{c}n \\ 3\end{array}\right)}=\lim _{n \rightarrow \infty} \frac{S_{n}}{3\left(\begin{array}{c}n \\ 3\end{array}\right)}+\frac{1}{3}=\lim _{n \rightarrow \infty} \frac{2 S_{n}}{n^{3}}+\frac{1}{3}$.

Next, we consider the relationship between the recurrences $S_{n}$ and $A_{n}$. Another (equivalent) way to write $S_{n}$ is:

$$
S_{n}=\max _{0 \leq i<n}\left\{S_{i}+n^{3} \cdot p_{3}\left(\frac{i}{n}\right)+n^{2} \cdot p_{2}\left(\frac{i}{n}\right)\right\},
$$

where $p_{3}(x)=\frac{x(1-x)^{2}}{2}$ and $p_{2}(x)=\frac{-x(1-x)}{2}$. Looking at $S_{n}$ defined in this way, we are tempted to extend it to a more general type of recurrence as follows. Let $k$ be a positive integer and let $p_{0}(x), p_{1}(x), \ldots, p_{k}(x)$ be polynomials such that $p_{d}(x)$ is a polynomial of degree $d$ for every $d \in\{0,1, \ldots, k\}$. Set $G_{0}=p_{0}(0)$, and for $n \geq 1$, define:

$$
G_{n}=\max _{0 \leq i<n}\left\{G_{i}+\sum_{d=0}^{k} n^{d} p_{d}\left(\frac{i}{n}\right)\right\} .
$$

Now, if we restrict the recurrence $G_{n}$ to the special case where $p_{d}(x)=0$ for all $d \in\{0,1, \ldots, k-1\}$ and $p_{k}(0)=0$, we obtain precisely the recurrence $A_{n}$.

\section{The Asymptotic Behavior of $S_{n}$ and $S_{n}^{\prime}$}

In order to analyze the asymptotic behavior of $S_{n} / n^{3}$, we define $s_{n}=S_{n} / n^{3}$ and rewrite $S_{n}$ in terms of $s_{n}$. This gives $s_{0}=0$, and for $n \geq 1$ :

$$
s_{n}=\max _{0 \leq i<n}\left\{s_{n, i}\right\} \text {, where } s_{n, i}=p_{3}\left(\frac{i}{n}\right)+\frac{1}{n} \cdot p_{2}\left(\frac{i}{n}\right)+s_{i} \cdot\left(\frac{i}{n}\right)^{3} .
$$

Here, $p_{3}$ and $p_{2}$ are the polynomials $p_{3}(x)=\frac{x(1-x)^{2}}{2}$ and $p_{2}(x)=\frac{-x(1-x)}{2}$, introduced in Section 2. Consider the function $\frac{p_{3}(x)}{1-x^{3}}$. It has a unique maximum value on the interval $[0,1)$. Call this value $\alpha$ and let $\beta$ be the point where $\alpha$ is obtained, i.e., $\frac{p_{3}(\beta)}{1-\beta^{3}}=\alpha$. By straightforward calculations, we have $\alpha=\frac{2 \sqrt{3}-3}{6}$, $\beta=\frac{\sqrt{3}-1}{2}$. In this section, we shall prove that $\lim _{n \rightarrow \infty} s_{n}=\alpha$.

First, we introduce two sequences $l_{n}, u_{n}(n \geq 0)$ and show that they provide a lower bound and an upper bound, respectively, on each term in the sequence $s_{n}$. Let $l_{0}=u_{0}=0$ and, for $n \geq 1$, define:

$$
\left\{\begin{array}{l}
l_{n}=\max _{0 \leq i<n}\left\{l_{n, i}\right\}, \quad \text { where } l_{n, i}=p_{3}\left(\frac{i}{n}\right)+\frac{1}{n} p_{2}\left(\frac{i}{n}\right)+\alpha\left(\frac{i}{n}-\frac{1}{n}\right)^{3}, \\
u_{n}=\max _{0 \leq i<n}\left\{u_{n, i}\right\}, \text { where } u_{n, i}=p_{3}\left(\frac{i}{n}\right)+\frac{1}{n} p_{2}\left(\frac{i}{n}\right)+\alpha\left(\frac{i}{n}\right)^{3} .
\end{array}\right.
$$

In the next four lemmas, we show that the following chain of inequalities holds for every integer $n \geq 1$ :

$$
\alpha\left(1-\frac{1}{n}\right)^{3} \leq l_{n} \leq s_{n} \leq u_{n} \leq \alpha .
$$


Lemma 3. For all $n \geq 0, u_{n} \leq \alpha$.

Proof. By the definition of $\alpha$, we have $\frac{p_{3}(x)}{1-x^{3}} \leq \alpha$, for $0 \leq x<1$. This yields $p_{3}(x)+\alpha x^{3} \leq \alpha$, for $0 \leq x<1$. Since $u_{n}$ is defined as $\max _{0 \leq i<n}\left\{p_{3}\left(\frac{i}{n}\right)+\frac{1}{n} p_{2}\left(\frac{i}{n}\right)+\right.$ $\left.\alpha\left(\frac{i}{n}\right)^{3}\right\}$ and $p_{2}(x) \leq 0$ for all $0 \leq x<1$, we have $u_{n} \leq \alpha$.

Lemma 4. For all $n \geq 0, s_{n} \leq u_{n}$.

Proof. By induction on $n$. For $n=0, u_{0}=s_{0}=0$. Next, suppose $s_{m} \leq u_{m}$ for all $m<n$. For each integer $0 \leq i<n$, by Lemma 3, we have $s_{n, i}-u_{n, i}=s_{i}\left(\frac{i}{n}\right)^{3}-$ $\alpha\left(\frac{i}{n}\right)^{3} \leq s_{i}\left(\frac{i}{n}\right)^{3}-u_{i}\left(\frac{i}{n}\right)^{3}=\left(s_{i}-u_{i}\right)\left(\frac{i}{n}\right)^{3} \leq 0$. Therefore, $s_{n}=\max _{1 \leq i<n}\left\{s_{n, i}\right\}=$ $\max _{1 \leq i<n}\left\{u_{n, i}+\left(s_{n, i}-u_{n, i}\right)\right\} \leq \max _{1 \leq i<n}\left\{u_{n, i}\right\}=u_{n}$.

Lemma 5. For all $n \geq 1, l_{n} \geq \alpha\left(1-\frac{1}{n}\right)^{3}$.

Proof. For $n \leq 15$, the inequality can be verified by computation. For $n \geq 16$, we show that $l_{n} \geq \alpha\left(1-\frac{1}{n}\right)^{3}$. First note that:

(*1) Since $\beta-\frac{1}{n} \leq \frac{\lfloor\beta n\rfloor}{n} \leq \beta=\frac{\sqrt{3}-1}{2}<\frac{1}{2}$ and $p_{2}(x)$ is decreasing on $\left[0, \frac{1}{2}\right]$, we have $p_{2}\left(\frac{\lfloor\beta n\rfloor}{n}\right) \geq p_{2}(\beta)$.

$\left({ }^{*} 2\right)$ We have $p_{3}(x) \geq p_{3}(\beta)$ for $x \in[0.302, \beta]$. For $n \geq 16, \frac{\lfloor\beta n\rfloor}{n}>\beta-\frac{1}{n} \geq$ $\beta-\frac{1}{16}>0.302$, therefore we have $p_{3}\left(\frac{\lfloor\beta n\rfloor}{n}\right)>p_{3}(\beta)$.

Then, it follows that:

$$
\begin{aligned}
& l_{n}-\alpha\left(1-\frac{1}{n}\right)^{3} \geq l_{n,\lfloor\beta n\rfloor}-\alpha\left(1-\frac{1}{n}\right)^{3} \\
= & p_{3}\left(\frac{\lfloor\beta n\rfloor}{n}\right) \underbrace{-p_{3}(\beta)+\alpha\left(1-\beta^{3}\right)}_{=0}+\frac{1}{n} p_{2}\left(\frac{\lfloor\beta n\rfloor}{n}\right)+\alpha\left(\left(\frac{\lfloor\beta n\rfloor}{n}-\frac{1}{n}\right)^{3}-\left(1-\frac{1}{n}\right)^{3}\right) \\
= & \underbrace{\left(\frac{\lfloor\beta n\rfloor}{n}\right)-p_{3}(\beta)}_{3}+\alpha\left(\left(\frac{\lfloor\beta n\rfloor}{n}-\frac{1}{n}\right)^{3}-\beta^{3}+1-\left(1-\frac{1}{n}\right)^{3}\right)+\frac{1}{n} p_{2}\left(\frac{\lfloor\beta n\rfloor}{n}\right) \\
\geq & \alpha\left(\left(\frac{\lfloor\beta n\rfloor}{n}-\frac{1}{n}\right)^{3}-\left(\beta-\frac{2}{n}\right)^{3}+\frac{3-6 \beta^{2}}{n}+\frac{12 \beta-3}{n^{2}}-\frac{7}{n^{3}}\right)+\frac{1}{n} \cdot \underbrace{p_{2}\left(\frac{\lfloor\beta n\rfloor}{n}\right)} \\
\geq & \alpha(\underbrace{\left(\left(\frac{\lfloor\beta n\rfloor}{n}-\frac{1}{n}\right)^{3}-\left(\beta-\frac{2}{n}\right)^{3}\right.}_{\geq 0})+\underbrace{\frac{\alpha}{n}\left(3-6 \beta^{2}+\frac{p_{2}(\beta)}{\alpha}+\frac{12 \beta-3}{n}+\frac{-7}{n^{2}}\right.}_{\geq 0, \text { for } n \geq 3}) \geq 0 .
\end{aligned}
$$

Lemma 6. For all $n \geq 1, s_{n} \geq l_{n}$.

Proof. By induction on $n$. For $n=0, s_{0}=l_{0}=0$. Next, suppose $s_{m} \geq l_{m}$, for all $m<n$. For each integer $0 \leq i<n$, by Lemma 5 , we have $s_{n, i}-l_{n, i}=$ $s_{i}\left(\frac{i}{n}\right)^{3}-\alpha\left(\frac{i}{n}-\frac{1}{n}\right)^{3}=s_{i}\left(\frac{i}{n}\right)^{3}-\alpha\left(1-\frac{1}{i}\right)^{3}\left(\frac{i}{n}\right)^{3} \geq\left(s_{i}-l_{i}\right)\left(\frac{i}{n}\right)^{3} \geq 0$. Therefore, $\max _{0 \leq i<n}\left\{s_{n, i}\right\} \geq \max _{0 \leq i<n}\left\{l_{n, i}\right\}$, which gives $s_{n} \geq l_{n}$. 
We now obtain the main result of this section:

Theorem 1. $\lim _{n \rightarrow \infty} \frac{S_{n}}{n^{3}}=\lim _{n \rightarrow \infty} s_{n}=\alpha=\frac{2 \sqrt{3}-3}{6} \approx 0.077350 \ldots$

Proof. By Lemmas 36, we have $\alpha\left(1-\frac{1}{n}\right)^{3} \leq s_{n} \leq \alpha$. Therefore, $\alpha=\lim _{n \rightarrow \infty} \alpha(1-$ $\left.\frac{1}{n}\right)^{3} \leq \lim _{n \rightarrow \infty} s_{n} \leq \alpha$, i.e., $\lim _{n \rightarrow \infty} s_{n}=\alpha$.

Finally, using Theorem 1 together with Lemma 2 gives:

Corollary 1. $\lim _{n \rightarrow \infty} \frac{S_{n}^{\prime}}{3\left(\begin{array}{c}n \\ 3\end{array}\right)}=\frac{2(\sqrt{3}-1)}{3} \approx 0.488033 \ldots$

Remark. Corollary 1 gives a strengthening of the inapproximability bound in Theorem 8 in 11; just change the " 0.4883 " to any real number strictly larger than $\frac{2(\sqrt{3}-1)}{3}$, for example "0.488034". Moreover, we can strengthen Lemma 5 in [2] (which says that $S_{n}^{\prime} / 3\left(\begin{array}{l}n \\ 3\end{array}\right)>0.4800$ ) and the resulting approximation ratio in Theorem 2 in [2] by observing that $S_{n}^{\prime} / 3\left(\begin{array}{c}n \\ 3\end{array}\right)=2 \cdot \frac{S_{n}}{n^{3}} \frac{n^{2}}{(n-1)(n-2)}+\frac{1}{3} \geq 2 \cdot \alpha \cdot\left(\frac{n-1}{n}\right)^{3}$. $\frac{n^{2}}{(n-1)(n-2)}+\frac{1}{3}$ by Lemmas 5 and 6, and then rewriting it as $2 \alpha \cdot \frac{(n-1)^{2}}{(n-2) n}+\frac{1}{3}>$ $2 \alpha+\frac{1}{3}=\frac{2(\sqrt{3}-1)}{3}$. In other words, $S_{n}^{\prime} / 3\left(\begin{array}{l}n \\ 3\end{array}\right)>\frac{2(\sqrt{3}-1)}{3} \approx 0.488033 \ldots$

\section{The Asymptotic Behavior of $A_{n}$}

The asymptotic behavior of $A_{n}$ depends on the properties of $p_{k}(x) /\left(1-x^{k}\right)$. We define $\alpha_{p}=\sup \left\{p_{k}(x) /\left(1-x^{k}\right): 0 \leq x<1\right\}$, when $\sup \left\{p_{k}(x) /\left(1-x^{k}\right): 0 \leq\right.$ $x<1\}<\infty 1$ There are four possible cases:

(C1) $\sup \left\{p_{k}(x) /\left(1-x^{k}\right): 0 \leq x<1\right\}=\infty$.

$(\mathrm{C} 2) \sup \left\{p_{k}(x) /\left(1-x^{k}\right): 0 \leq x<1\right\}=\alpha_{p}<\infty$, and $\lim _{x \rightarrow 1^{-}} \frac{p_{k}(x)}{1-x^{k}}=\alpha_{p}$.

(C3) $\sup \left\{p_{k}(x) /\left(1-x^{k}\right): 0 \leq x<1\right\}=\alpha_{p}=0$, and $\frac{p_{k}(0)}{1-0^{k}}=\alpha_{p}=0$.

(C4) $\sup \left\{p_{k}(x) /\left(1-x^{k}\right): 0 \leq x<1\right\}=\alpha_{p}<\infty$, and there exists a $\beta_{p}$, where $0<\beta_{p}<1$, such that $\frac{p_{k}\left(\beta_{p}\right)}{1-\beta_{p}^{k}}=\alpha_{p}$.

The definition of $A_{n}$ is $\max _{0 \leq i<n}\left\{n^{k} p_{k}\left(\frac{i}{n}\right)+A_{i}\right\}$, for $n>0$. If we substitute $A_{n}$ $(m-1)$ times recursively, we get

$$
\begin{aligned}
A_{n} & =\max _{0 \leq i_{2}<i_{1}<n}\left\{n^{k} p_{k}\left(\frac{i_{1}}{n}\right)+i_{1}^{k} p_{k}\left(\frac{i_{2}}{i_{1}}\right)+A_{i_{2}}\right\}=\cdots \\
& =\max _{0 \leq i_{m}<\cdots<i_{1}<i_{0}}\left\{\sum_{t=0}^{m-1} i_{t}^{k} p_{k}\left(\frac{i_{t+1}}{i_{t}}\right)+A_{i_{m}}\right\} .
\end{aligned}
$$

${ }^{1}$ Note that we use "sup" instead of "max" for the following reason. For some $p_{k}(x)$, e.g., $k=3, p_{3}(x)=-x^{3}+x$, there is no maximum value for $\frac{p_{k}(x)}{1-x^{k}}, 0 \leq x<1$. However, there exists an upper bound for $\frac{p_{k}(x)}{1-x^{k}}, 0 \leq x<1$. 
By choosing $i_{t}=n-t$, we define $L_{n}$ with $L_{0}=0$, and for $n \geq 1$,

$$
L_{n}=n^{k} p_{k}\left(\frac{n-1}{n}\right)+L_{n-1} .
$$

We substitute $L_{n}(m-1)$ times, which gives: $L_{n}=\sum_{t=0}^{n-1}(n-t)^{k} p_{k}\left(\frac{n-t-1}{n-t}\right)$.

Since $A_{n}$ is taking the maximum value among all parameters $\left\{i_{t}\right\}$, we have $A_{n} \geq L_{n}$. For case (C1), we show that $\lim _{n \rightarrow \infty} \frac{L_{n}}{n^{k}}=\infty$ in Lemma 7 It follows that $\lim _{n \rightarrow \infty} \frac{A_{n}}{n^{k}}=\infty$. For case (C2), we show that $\lim _{n \rightarrow \infty} \frac{L_{n}}{n^{k}}=\alpha_{p}$ and $A_{n}$ also has an upper bound $\alpha_{p}$. Therefore, $\lim _{n \rightarrow \infty} \frac{A_{n}}{n^{k}}=\alpha_{p}$.

Lemma 7. If $\sup \left\{\frac{p_{k}(x)}{1-x^{k}}: 0 \leq x<1\right\}=\infty$, then $\lim _{n \rightarrow \infty} \frac{A_{n}}{n^{k}}=\infty$.

Proof. Assume that $p_{k}(x)=\sum_{i=1}^{k} c_{i} x^{i}$. We observe that $(n-t)^{k} p_{k}\left(\frac{n-t-1}{n-t}\right)$ is a polynomial of $(n-t)$ with degree at most $k$. Furthermore, the coefficient of $(n-t)^{k}$ in $(n-t)^{k} p_{k}\left(\frac{n-t-1}{n-t}\right)=\sum_{i=1}^{k} c_{i}(n-t-1)^{i}(n-t)^{k-i}$ equals $\sum_{i=1}^{k} c_{i}=p_{k}(1)$. For the reason that $\lim _{x \rightarrow 1^{-}} \frac{p_{k}(x)}{1-x^{k}}=\infty$, we have $p_{k}(1)>0$.

Since $L_{n}=\sum_{t=0}^{n-1}(n-t)^{k} p_{k}\left(\frac{n-t-1}{n-t}\right), L_{n}$ is a polynomial of $n$ with degree $k+1$. Therefore, $\lim _{n \rightarrow \infty} \frac{A_{n}}{n^{k}} \geq \lim _{n \rightarrow \infty} \frac{L_{n}}{n^{k}}=\infty$.

Lemma 8. If $\sup \left\{\frac{p_{k}(x)}{1-x^{k}}: 0 \leq x<1\right\}=\alpha_{p}<\infty$ and $\lim _{x \rightarrow 1^{-}} \frac{p_{k}(x)}{1-x^{k}}=\alpha_{p}$, then $\lim _{n \rightarrow \infty} \frac{A_{n}}{n^{k}}=\alpha_{p}$

Proof. The proof of the upper bound of $A_{n}$ is at most $\alpha_{p}$ is similar to that of Lemma 4.

Assume that $p_{k}(x)=\sum_{i=1}^{k} c_{i} x^{i}$. The coefficient of $(n-t)$ in $(n-t)^{k} p_{k}\left(\frac{n-t-1}{n-t}\right)$ equals $p_{k}(1)$. However, for the reason that $\lim _{x \rightarrow 1^{-}} \frac{p_{k}(x)}{1-x^{k}}=\alpha_{p}$, we have $p_{k}(1)=0$. Hence, $L_{n}$ is a polynomial with degree at most $k$.

Furthermore, the coefficient of $(n-t)^{k-1}$ in $(n-t)^{k} p_{k}\left(\frac{n-t-1}{n-t}\right)=\sum_{i=1}^{k} c_{i}(n-$ $t-1)^{i}(n-t)^{k-i}$ is $\sum_{i=1}^{k}-i c_{i}=-p_{k}^{\prime}(1)$. We have the coefficient of $n^{k}$ in $L_{n}$ equals that in $\sum_{t=0}^{n-1}-p_{k}^{\prime}(1) \cdot(n-t)^{k-1}$. Then the coefficient of $n^{k}$ in $L_{n}$ equals $\frac{-p_{k}^{\prime}(1)}{k}$.

Since $(x-1)$ is a factor of $p_{k}(x)$, let $q_{k}(x)=\frac{p_{k}(x)}{x-1}$. Then $\frac{d}{d x} p_{k}(x)=\frac{d}{d x}\left(q_{k}(x)\right.$ $(x-1))=q_{k}(x)+(x-1) \frac{d}{d x}\left(q_{k}(x)\right)$. Hence, $p_{k}^{\prime}(1)=q_{k}(1)$. Moreover,

$$
\alpha_{p}=\lim _{x \rightarrow 1^{-}} \frac{p_{k}(x)}{1-x^{k}}=\lim _{x \rightarrow 1^{-}} \frac{(x-1) q_{k}(x)}{1-x^{k}}=\lim _{x \rightarrow 1^{-}} \frac{-q_{k}(x)}{1+x+\cdots+x^{k-1}}=\frac{-q_{k}(1)}{k} .
$$


Finally, we have $\lim _{n \rightarrow \infty} \frac{L(n)}{n^{k}}=\frac{-p_{k}^{\prime}(1)}{k}=\frac{-q_{k}(1)}{k}=\alpha_{p}$. Then, $\lim _{n \rightarrow \infty} \frac{A_{n}}{n^{k}}=\alpha_{p}$.

Lemma 9. If $\sup \left\{\frac{p_{k}(x)}{1-x^{k}}: 0 \leq x<1\right\}=0$, then $A_{n}=0$.

Proof. By induction on $n$. For $n=0$, it holds that $A_{0}=0$. Next, suppose that $A_{m}=0$ for all $m<n$. Then, since $\alpha_{p}=0$, we have $p_{k}(x) \leq 0$, for $0 \leq x \leq 1$, therefore

$$
A_{n}=\max _{0 \leq i<n}\left\{n^{k} p_{k}\left(\frac{i}{n}\right)+A_{i}\right\} \leq \max _{0 \leq i<n}\left\{A_{i}\right\}=0 .
$$

To study the asymptotic value of $A_{n} / n^{k}$ in case (C4), we define $a_{n}=A_{n} / n^{k}$, and rewrite the recurrence for $A_{n}$ in terms of $a_{n}$ as follows. Let $a_{0}=0$ and, for $n \geq 1$,

$$
a_{n}=\max _{0 \leq i<n}\left\{a_{n, i}\right\}, \text { where } a_{n, i}=p_{k}\left(\frac{i}{n}\right)+a_{i}\left(\frac{i}{n}\right)^{k} .
$$

To find a lower bound of $a_{n}$, we rewrite $a_{n}$ by recursively substituting it $(m-1)$ times, for some value of $m$ to be specified later.

$$
\begin{aligned}
a_{n} & =\max _{0 \leq i_{1}<n}\left\{p_{k}\left(\frac{i_{1}}{n}\right)+\left(\frac{i_{1}}{n}\right)^{k} a_{i_{1}}\right\}=\max _{0 \leq i_{2}<i_{1}<n}\left\{p_{k}\left(\frac{i_{1}}{n}\right)+\left(\frac{i_{1}}{n}\right)^{k}\left(p_{k}\left(\frac{i_{2}}{i_{1}}\right)+\left(\frac{i_{2}}{i_{1}}\right)^{k} a_{i_{2}}\right)\right\} \\
& =\cdots=\max _{0 \leq i_{m}<\cdots<i_{1}<i_{0}=n}\left\{\left(\sum_{t=0}^{m-1}\left(\frac{i_{t}}{n}\right)^{k} p_{k}\left(\frac{i_{t+1}}{i_{t}}\right)\right)+\left(\frac{i_{m}}{n}\right)^{k} a_{i_{m}}\right\} .
\end{aligned}
$$

By choosing $i_{t}=\left\lfloor\beta_{p}^{t} n\right\rfloor$ for $a_{n}$, we define $l_{n, m}=\left(\sum_{t=0}^{m-1}\left(\frac{\left\lfloor\beta_{p}^{t} n\right\rfloor}{n}\right)^{k} p_{k}\left(\frac{\left\lfloor\beta_{p}^{t+1} n\right\rfloor}{\left[\beta_{p}^{t} n\right\rfloor}\right)\right)+$ $\left(\frac{\left\lfloor\beta_{p}^{m} n\right\rfloor}{n}\right)^{k} a_{i_{m}}$. For the condition that $\left\lfloor\beta_{p}^{t-1} n\right\rfloor>\left\lfloor\beta_{p}^{t} n\right\rfloor$ with $t<m$ to hold, we need $m$ to satisfy $\beta_{p}^{m-1} n \geq \beta_{p}^{m} n+1$, i.e., $n>\frac{1}{\beta_{p}^{m-1}\left(1-\beta_{p}\right)}$. Since $a_{n}$ is taking the maximum value among all parameters $\left\{i_{t}\right\}$, we have $a_{n} \geq l_{n, m}$.

To show that $l_{n, m}$ converges to $\alpha_{p}$, we replace $\alpha_{p}$ by $p_{k}\left(\beta_{p}\right)+\alpha_{p} \beta_{p}^{k}(m-1)$ times and find an expression for $\alpha_{p}$ which looks similar to the formula for $l_{n, m}$.

$$
\begin{aligned}
\alpha_{p} & =p_{k}\left(\beta_{p}\right)+\alpha_{p} \beta^{k}=p_{k}\left(\beta_{p}\right)+\beta_{p}^{k}\left(p_{k}\left(\beta_{p}\right)+\alpha_{p} \beta_{p}^{k}\right)=\cdots \\
& =\left(\sum_{t=0}^{m-1} \beta_{p}^{t k} p_{k}\left(\beta_{p}\right)\right)+\beta_{p}^{m k} \alpha_{p} .
\end{aligned}
$$

In the next lemma, we show that $l_{n, m}$ is close to $\alpha_{p}$ based on two observations: (1) $\beta_{p}^{m k} \alpha_{p}$ is very small for sufficiently large $m$; and (2) when $\left\lfloor\beta_{p}^{t} n\right\rfloor$ is large, $\frac{\left\lfloor\beta_{p}^{t+1} n\right\rfloor}{\left\lfloor\beta_{p}^{t} n\right\rfloor}$ is close to $\beta_{p}$ and then we have $\beta_{p}^{t k} p_{k}\left(\beta_{p}\right)$ is close to $\left(\frac{\left\lfloor\beta_{p}^{t} n\right\rfloor}{n}\right)^{k} p_{k}\left(\frac{\left\lfloor\beta_{p}^{t+1} n\right\rfloor}{\left\lfloor\beta_{p}^{t} n\right\rfloor}\right)$.

Lemma 10. If $\sup \left\{\frac{p_{k}(x)}{1-x^{k}}: 0 \leq x<1\right\}=\alpha_{p}<\infty$ and there exists a $\beta_{p}$, where $0<\beta_{p}<1$, such that $\frac{p_{k}\left(\beta_{p}\right)}{1-\beta_{p}^{k}}=\alpha_{p}$, then $\lim _{n \rightarrow \infty} \frac{A_{n}}{n^{k}}=\alpha_{p}$.

Proof. The proof of $\alpha_{p}$ being the upper bound of $a_{n}$ is similar to that of Lemma 4 To pave the way for the lower bound of $a_{n}$, we introduce two notations $M_{1}$ and $M_{2}$. 
Consider the Taylor series expansion for $p_{k}(x)$ in $\beta_{p}: p_{k}(x)=p_{k}\left(\beta_{p}\right)+$ $\sum_{i=1}^{k} \frac{f^{(i)}\left(\beta_{p}\right)}{i !}\left(x-\beta_{p}\right)^{i}$. For $0 \leq x<1$, we have

$$
\begin{aligned}
\left|p_{k}(x)-p_{k}\left(\beta_{p}\right)\right| & \leq\left(x-\beta_{p}\right) \sum_{i=1}^{k}\left|\frac{p_{k}^{(i)}\left(\beta_{p}\right)}{i !}\left(x-\beta_{p}\right)^{i-1}\right| \\
& \left.\leq\left(x-\beta_{p}\right) \sum_{i=1}^{k}\left|\frac{p_{k}^{(i)}\left(\beta_{p}\right)}{i !}\right| \quad \text { (because } 0<x, \beta_{p}<1\right) \\
& \leq\left(x-\beta_{p}\right) M_{1}, \quad \text { where } M_{1}=\sum_{i=1}^{k}\left|\frac{p_{k}^{(i)}\left(\beta_{p}\right)}{i !}\right| .
\end{aligned}
$$

Since $p_{k}(x)$ is a polynomial, there exists a maximum value of $p_{k}(x)$ on the interval $[0,1]$. Let

$$
M_{2}=\max _{0 \leq x \leq 1}\left\{p_{k}(x)\right\} .
$$

Furthermore, for the reason that $0<\beta<1$, we have:

$$
\begin{gathered}
\beta_{p}^{t k}\left(\beta_{p}-\frac{\left\lfloor\beta_{p}^{t+1} n\right\rfloor}{\left\lfloor\beta_{p}^{t} n\right\rfloor}\right) \leq \frac{\beta_{p}^{t k}}{\left\lfloor\beta_{p}^{t} n\right\rfloor} \leq \frac{2 \beta_{p}^{t k}}{\beta_{p}^{t} n} \leq \frac{2 \beta_{p}^{t(k-1)}}{n} \leq \frac{2}{n}, \text { and } \\
\beta_{p}^{t k}-\left(\frac{\left\lfloor\beta_{p}^{t} n\right\rfloor}{n}\right)^{k}=\left(\beta_{p}^{t}-\frac{\left\lfloor\beta_{p}^{t} n\right\rfloor}{n}\right) \sum_{i=0}^{k-1}\left(\left(\beta_{p}^{t}\right)^{i}\left(\frac{\left\lfloor\beta_{p}^{t} n\right\rfloor}{n}\right)^{k-1-i}\right) \leq \frac{k}{n} .
\end{gathered}
$$

Since $M_{1}, M_{2}, \alpha_{p}$ and $\beta_{p}$ are fixed values, for all $\epsilon>0$, there exists a positive integer $m$ such that:

$$
\beta_{p}^{m k}\left(2 m M_{1}+k m M_{2}+\alpha_{p}\right)<\epsilon .
$$

For $n \geq \max \left\{\left\lceil\frac{1}{\beta_{p}^{m k}}\right\rceil,\left\lceil\frac{1}{\beta_{p}^{m-1}\left(1-\beta_{p}\right)}\right\rceil\right\}$, we have

$$
\begin{aligned}
& \left|\alpha_{p}-a_{n}\right| \\
\leq & \left.\left|\alpha_{p}-l_{n, m}\right| \quad \text { (because } l_{n, m} \leq a_{n} \leq \alpha\right) \\
\leq & \left|\sum_{t=0}^{m-1}\left(\beta_{p}^{t k} f\left(\beta_{p}\right)-\left(\frac{\left\lfloor\beta_{p}^{t} n\right\rfloor}{n}\right)^{k} p_{k}\left(\frac{\left\lfloor\beta_{p}^{t+1} n\right\rfloor}{\left[\beta_{p}^{t} n\right]}\right)\right)\right|+\left|\beta_{p}^{m k} \alpha_{p}-\left(\frac{\left\lfloor\beta_{p}^{m} n\right\rfloor}{n}\right)^{k} a_{m}\right| \\
\leq & \left|\sum_{t=0}^{m-1}(\beta_{p}^{t k} p_{k}\left(\beta_{p}\right) \underbrace{-\beta_{p}^{t k} p_{k}\left(\frac{\left\lfloor\beta_{p}^{t+1} n\right\rfloor}{\left\lfloor\beta_{p}^{t} n\right\rfloor}\right)+\beta_{p}^{t k} p_{k}\left(\frac{\left\lfloor\beta_{p}^{t+1} n\right\rfloor}{\left\lfloor\beta_{p}^{t} n\right\rfloor}\right)}_{=0}-\left(\frac{\left[\beta_{p}^{t} n\right]}{n}\right)^{k} p_{k}\left(\frac{\left\lfloor\beta_{p}^{t+1} n\right\rfloor}{\left\lfloor\beta_{p}^{t} n\right\rfloor}\right))\right| \\
& +\beta_{p}^{m k} \alpha_{p}
\end{aligned}
$$




$$
\begin{aligned}
& =\sum_{t=0}^{m-1}\left|\beta_{p}^{t k}\left(p_{k}\left(\beta_{p}\right)-p_{k}\left(\frac{\left\lfloor\beta_{p}^{t+1} n\right\rfloor}{\left\lfloor\beta_{p}^{t} n\right\rfloor}\right)\right)+\left(\beta_{p}^{t k}-\left(\frac{\left\lfloor\beta_{p}^{t} n\right\rfloor}{n}\right)^{k}\right) p_{k}\left(\frac{\left\lfloor\beta_{p}^{t+1} n\right\rfloor}{\left\lfloor\beta_{p}^{t} n\right\rfloor}\right)\right|+\beta_{p}^{m k} \alpha_{p} \\
& \leq \sum_{t=0}^{m-1}\left(\left|\beta_{p}^{t k}\left(\beta_{p}-\frac{\left\lfloor\beta_{p}^{t+1} n\right\rfloor}{\left\lfloor\beta_{p}^{t} n\right\rfloor}\right) M_{1}\right|+\left|\left(\beta_{p}^{t k}-\left(\frac{\left\lfloor\beta_{p}^{t} n\right\rfloor}{n}\right)^{k}\right) M_{2}\right|\right)+\beta_{p}^{m k} \alpha_{p} \quad \text { (by (1) (12) ) } \\
& \leq \frac{1}{n} \sum_{t=0}^{m-1}\left|2 M_{1}+k M_{2}\right|+\beta_{p}^{m k} \alpha_{p} \quad(\text { by (3) (4) (4) }) \\
& \leq \beta_{p}^{m k}\left(m\left(2 M_{1}+k M_{2}\right)+\alpha_{p}\right) \leq \epsilon \quad\left(\text { by } n \geq\left\lceil\frac{1}{\beta_{p}^{m k}}\right\rceil \text { and (5) }\right)
\end{aligned}
$$

Combining Lemmas 7-10, we obtain the following result.

Theorem 2. $\lim _{n \rightarrow \infty} \frac{A_{n}}{n^{k}}=\sup \left\{\frac{p_{k}(x)}{1-x^{k}}: 0 \leq x<1\right\}$.

Remark. When we take $k=3$ and $p_{3}(x)=\frac{x(1-x)(1-x)}{2}$ in $A_{n}$, we have $\lim _{n \rightarrow \infty} A_{n} / n^{3}=(2 \sqrt{3}-3) / 6$, which is equal to $\lim _{n \rightarrow \infty} S_{n} / n^{3}$. We can see that the term $p_{2}(x)$ in $S_{n}$ has no effect on the asymptotic behavior of $S_{n}$.

\section{Concluding Remarks}

We note that to analyze minimization recurrences analogous to $A_{n}$, we can apply our technique from Section 4 as follows. Suppose that $B_{n}=\min _{0 \leq i<n}\left\{n^{k} p_{k}\left(\frac{i}{n}\right)+B_{i}\right\}$. Let $A_{n}=-B_{n}$. Then $A_{n}=\max _{0 \leq i<n}\left\{n^{k} \cdot\left(-p_{k}\left(\frac{i}{n}\right)\right)+A_{i}\right\}$, and Theorem 2 gives:

Corollary 2. $\lim _{n \rightarrow \infty} \frac{B_{n}}{n^{k}}=\inf \left\{\frac{p_{k}(x)}{1-x^{k}}: 0 \leq x<1\right\}$.

We conclude this paper by mentioning two open problems. First, to derive a closed-form expression for the exact value of $S_{n}$ or to determine that such a formula does not exist is an open problem. Second, for the general case of $G_{n}$ (see Section 2), we can set $g_{n}=G_{n} / n^{k}$ and rewrite the recurrence relation as:

$$
g_{n}=\max _{0 \leq i<n}\left\{\left(\sum_{d=0}^{k} \frac{1}{n^{k-d}} p_{d}\left(\frac{i}{n}\right)\right)+g_{i}\left(\frac{i}{n}\right)^{k}\right\} .
$$

For $d<k$, the term $p_{d}\left(\frac{i}{n}\right)$ is multiplied by $\frac{1}{n^{k-d}}$. For sufficiently large $n$, the part $p_{d}\left(\frac{i}{n}\right)$ has a small effect on $g_{n}$, for $d<k$. Hence, we conjecture that the asymptotic behavior of $g_{n}$ is the same as that of $a_{n}$.

Acknowledgements. An-Chiang Chu was supported in part by the 2011 Summer Visiting Program from the Interchange Association, Japan. An-Chiang Chu and Kun-Mao Chao were supported in part by NSC grants 98-2221-E-002-081MY3 and 100-2221-E-002-131-MY3 from the National Science Council, Taiwan. Jesper Jansson and Richard S. Lemence were funded by the Special Coordination Funds for Promoting Science and Technology, Japan. 


\section{References}

1. Bininda-Emonds, O.R.P.: The evolution of supertrees. Trends in Ecology and Evolution 19(6), 315-322 (2004)

2. Byrka, J., Gawrychowski, P., Huber, K.T., Kelk, S.: Worst-case optimal approximation algorithms for maximizing triplet consistency within phylogenetic networks. Journal of Discrete Algorithms 8(1), 65-75 (2010)

3. Cormen, T., Leiserson, C., Rivest, R., Stein, C.: Introduction to Algorithms, 3rd edn. The MIT Press, Massachusetts (2009)

4. Felsenstein, J.: Inferring Phylogenies. Sinauer Associates, Inc., Sunderland (2004)

5. Fredman, M.L., Knuth, D.E.: Recurrence relations based on minimization. Journal of Mathematical Analysis and Applications 48(2), 534-559 (1974)

6. Ga̧sieniec, L., Jansson, J., Lingas, A., Östlin, A.: On the complexity of constructing evolutionary trees. Journal of Combinatorial Optimization 3(2-3), 183-197 (1999)

7. Gusfield, D., Eddhu, S., Langley, C.: Efficient reconstruction of phylogenetic networks with constrained recombination. In: Proceedings of the Computational Systems Bioinformatics Conference (CSB2 2003), pp. 363-374 (2003)

8. Henzinger, M.R., King, V., Warnow, T.: Constructing a tree from homeomorphic subtrees, with applications to computational evolutionary biology. Algorithmica 24(1), 1-13 (1999)

9. Hwang, H.-K., Tsai, T.-H.: An asymptotic theory for recurrence relations based on minimization and maximization. Theoretical Computer Science 290(3), 1475-1501 (2003)

10. Huson, D.H., Rupp, R., Scornavacca, C.: Phylogenetic Networks: Concepts, Algorithms and Applications. Cambridge University Press (2010)

11. Jansson, J., Nguyen, N., Sung, W.: Algorithms for combining rooted triplets into a galled phylogenetic network. SIAM Journal on Computing 35(5), 1098-1121 (2006)

12. Kapoor, S., Reingold, E.M.: Recurrence relations based on minimization and maximization. Journal of Mathematical Analysis and Applications 109(2), 591-604 (1985)

13. Li, Z., Reingold, E.M.: Solution of a divide-and-conquer maximin recurrence. SIAM Journal on Computing 18(6), 1188-1200 (1989)

14. Morrison, D.: Introduction to Phylogenetic Networks. RJR Productions (2011)

15. Saha, A., Wagh, M.D.: Minmax recurrences in analysis of algorithms. In: Proceedings of Southeastcon 1993. IEEE (1993)

16. Wang, L., Ma, B., Li, M.: Fixed topology alignment with recombination. Discrete Applied Mathematics 104(1-3), 281-300 (2000) 